\title{
Erratum to: Immnopathology
}

\author{
Cheng Sun, Haoyu Sun, and Zhigang Tian
}

\section{Erratum to:}

Chapter 5 in: X.-H. Gao, H.-D. Chen (eds.), Practical Immunodermatology, DOI 10.1007/978-94-024-0902-4_5

The original version of this chapter unfortunately contained a mistake. The presentation of Fig. 5.2 was incorrect. Please refer next page (E2) for the corrected image.

The updated online version of the original chapter can be found under DOI 10.1007/978-94-024-0902-4 5

C. Sun $\bullet$ H. Sun

Institute of Immunology, School of Life Sciences and Medical

Center, University of Science and Technology of China, Hefei, China

Z. Tian $(\bowtie)$

Institute of Immunology, School of Life Sciences and Medical

Center, University of Science and Technology of China, Hefei,

China

e-mail: tzg@ustc.edu.cn 


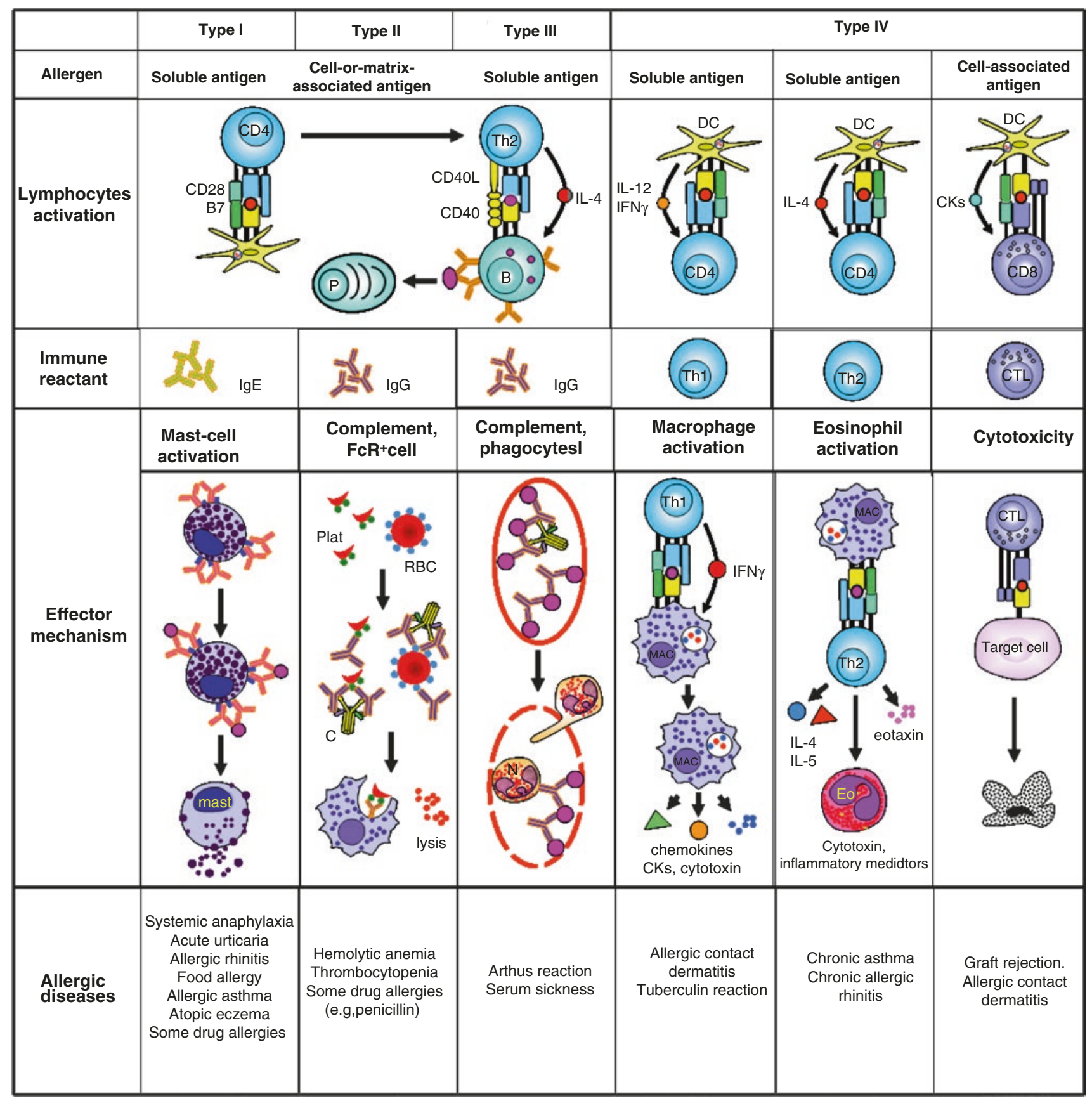

The original chapter has been corrected. 